\title{
The Third Superharmonic Resonance Analysis of RLC Circuit with Sensor Electronic Components
}

\author{
Gao-Feng LI ${ }^{\mathrm{a}}$ \\ Tangshan university, Department of Basic, China \\ ligaofeng0315@163.com
}

Keywords: Displacement sensor, Electronic components, RLC circuit, Nonlinear capacitance.

\begin{abstract}
Based on RLC series circuit of sensor electronic components with nonlinear capacitance research object, the first approximate solution of third superharmonic resonance of the nonlinear vibration system is obtained by means of the method of multiple scales for nonlinear oscillations. The third superharmonic resonance response curves of RLC series circuit have jump phenomenon and hysteresis phenomenon. With the increasing of resistance for all kinds of electronic components, the amplitude and resonant region of the primary resonance decrease. With the increasing of electromotive force (emf), the amplitude and resonant region of the primary resonance increase. The conclusion is provided a reference for design and manufacture of electronic components.
\end{abstract}

\section{Introduction}

Sensors are widely used in aviation, weapons industry and civil industry. In the aspect of civilian in dustry, sensors are widely used in the study of vibration detection and vibration of all kinds of large motor, air compressor, machine tools, vehicles, sleeper vibration table, chemical equipment, all kin ds of water pipelines, gas pipelines, bridges, buildings, etc. Capacitive displacement sensor consists of a RLC circuit and spring, plate and other elastomer coupling system dynamic model. Component $\mathrm{S}$ will present the nonlinear properties, while components are working in the big voltage or electric. So all circuit are nonlinear, such as electrical equipment, transformer coils, electronic control techno logy in the demodulation circuit and electronic devices in the highly nonlinear logic circuit, etc. The vibration of the capacitor is nonlinear, while circuit of capacitor plate vibrates under the action of el ectric field force. RLC circuit system has abundant dynamic phenomena, so grasps the nonlinear ch aracteristic of the rich in the circuit system can provide a reference basis to the development of the $\mathrm{s}$ ensor itself.

To study the nonlinear vibration, Yu-Min XIANG take the vibration equation of the solution and the accurate form of cycle. Functions show the nonlinear vibration characteristics of amplitude[1]. Zhong-Yu YAO use Lagrange equation model to analyze the transient process of RLC circuit[2]. Shi-Chang ZHAN consists a RLC series circuit with tungsten filament bulbs, transformer coil and capacitance[3]. M.Y.YOUNIS and A.H.NAYFEH use multi-scale method is to study the resonant micro beam in nonlinear response problem in electric field[4]. Qing-Yi YANG study nonlinear quantum effect of mesoscopic circuit, the RLC series circuit model is proposed for nonlinear resistance, and volt-ampere characteristic of diode as an example to analyze nonlinear resistance on the impact of the fluctuation[5]. Hai-Ning SUN analyzed a nonlinear RLC series circuit, inspired by square wave power supply circuit containing a core inductor, under appropriate conditions will appear chaotic phenomenon[6]. AliOksasogl studied RLC circuit system , and it can produce chaos phenomena under certain conditions[7]. S. k. Chakravarthy studied the circuit of nonlinear vibration and the parameters of the system. J. r. Marti and a. c. Soudack. using the ritz method to prove for A class of RLC circuit has the fundamental frequency vibration and chaotic phenomena[8]. Zhi-An YANG studied the parallel of RLC circuit including RLC circuit spring coupling system and RLC series circuit and micro beam coupling nonlinear vibration of the system[9-11]. RLC circuit system has abundant dynamic phenomena, grasps the nonlinear characteristic of the rich in the circuit system and the relationship between the electronic components, for the development of electronic components in itself can provide a reference basis. 
Based on the electromechanical coupling dynamics, the mathematical model of the RLC circuit composed of sensors is established, and considering the characteristics of the sensor itself. With the vibration equation of the RLC circuit as the research object, the application of nonlinear vibration theory and modern analysis method to solve the equation of [12]. The influence of system parameters on the nonlinear vibration characteristics with sensor are studied. The conclusions are provided as reference for the development of electronic components in the safety, reliability design and sensitivity design.

\section{Nonlinear Vibration Analysis of RLC Circuit Composed of Sensor}

The circuit of electronic components can be simplified as resistance $R$, inductance $L$, capacitance $C$ and power supply $E_{m}$ series, capacitance in circuit is nonlinear capacitance as figure 1 , let us use the approximation $u=\frac{1}{C_{0}} q+k_{2} q^{2}+k_{3} q^{3}+k_{4} q^{4}+\cdots$ where $C_{0}$ is the value of the "linear" capacitance, and $q$ is charge, and $k_{2}, k_{3}, k_{4}$ are the nonlinearity coefficients depending on the type of the sensor used.

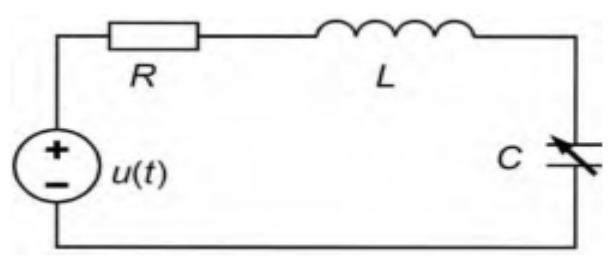

Figure 1. Nonlinear RLC series circuit

In this case, the differential equation governing the circuit's behavior of sensor is reduced to the equation of motion of the Duffing type oscillator:

$\ddot{q}+2 \mu \dot{q}+\omega_{0}^{2} q+\alpha_{2} q^{2}+\alpha_{3} q^{3}=f \cos (\omega t)$

where $\omega_{0}^{2}=\frac{1}{L C_{0}}, \alpha_{2}=\frac{k_{2}}{L}, \alpha_{3}=\frac{k_{3}}{L}, \mu=\frac{R}{2 L}, f=\frac{E_{m}}{L}$ 。

In this section the third superharmonic resonance of the sensor is investigated. the detuning parameter $\sigma$ is introduced according to the following from $3 \omega=\omega_{0}+\varepsilon \sigma, \sigma=o(1)$.

The method of multiple scales is applied to solve nonlinear vibration for the third superharmonic resonance for the first approximation

$\alpha(t)=a(\varepsilon t) \cos (3 \omega t-\varphi(\varepsilon t))+\frac{f}{\omega_{0}^{2}-\omega^{2}} \cos \omega t$

The amplitude frequency response equation of the third superharmonic resonance is

$\left[\mu^{2}+\left(\sigma-\frac{3 \alpha_{3} B^{2}}{\omega_{0}}-\frac{3 \alpha_{3}}{8 \omega_{0}} a^{2}\right)^{2}\right] a^{2}=\left(\frac{\alpha_{3} B^{3}}{\omega_{0}}\right)^{2}$

Solving this equation for $\sigma$ in terms of $a$ yields

$\sigma=\frac{3 \alpha_{3} B^{2}}{\omega_{0}}+\frac{3 \alpha_{3}}{8 \omega_{0}} a^{2} \pm \sqrt{\left(\frac{\alpha_{3} B^{3}}{\omega_{0} a}\right)^{2}-\mu^{2}}$

Therefore when $3 \omega \approx \omega_{0}$, the free-oscillation term does not decay to zero in spite the presence of damping. Moreover the nonlinearity adjusts the frequency of the free-oscillation term to exactly three times the frequency of the excitation so that the response is periodic.

Superharmonic resonance amplitude frequency response amplitude frequency response is very similar, with the main jump phenomena. Formant for the amplitude frequency response of the 
superharmonic resonance is very similar to that of the primary resonance amplitude. The resonance

peak for

$$
a_{\max }=\frac{\omega_{0} B^{3}}{\mu}=\frac{\omega_{0} f^{3}}{8 \mu\left(\omega_{0}^{2}-\omega^{2}\right)^{3}} .
$$

The resonance peak is related to the nonlinearity of the system, which is significantly different from that of the primary resonance.

\section{The Influence Law of Instance Analysis of System Parameters}

The sensor parameters are: $R=1 \Omega, E_{m}=4.5 \mathrm{~V}, L=0.6 \mathrm{H}, C=0.05$ 。 In this section, numerical results of the third superharmonic resonance are presented based on equation (3). Effects of the different parameter on the steady state response are discussed.

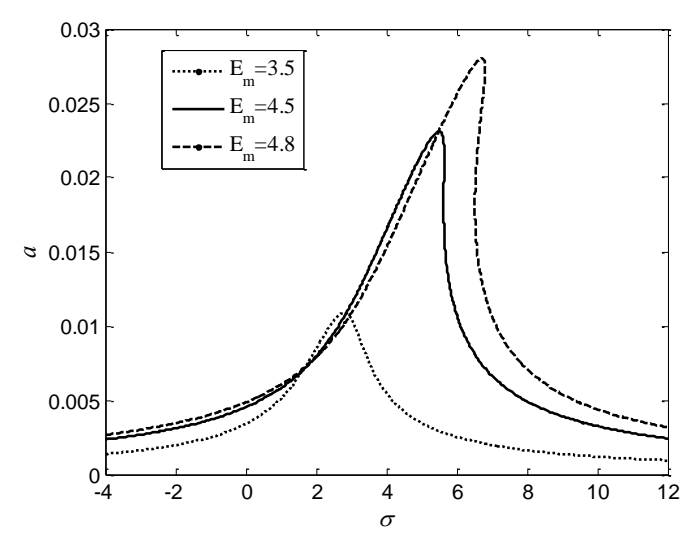

(a)

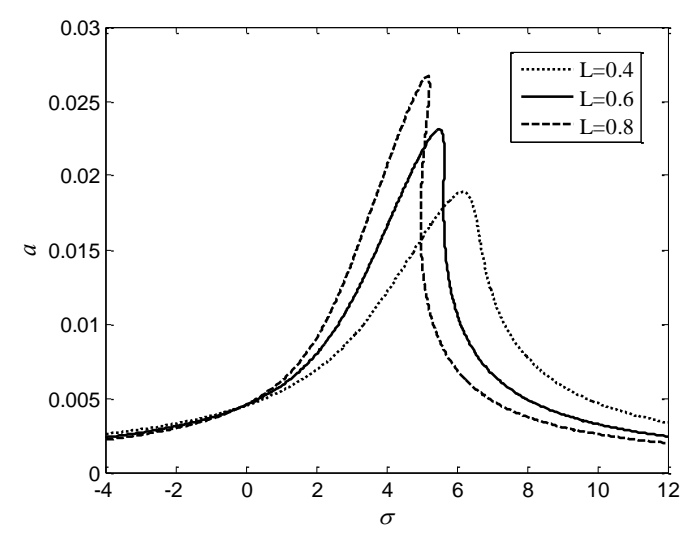

(c)

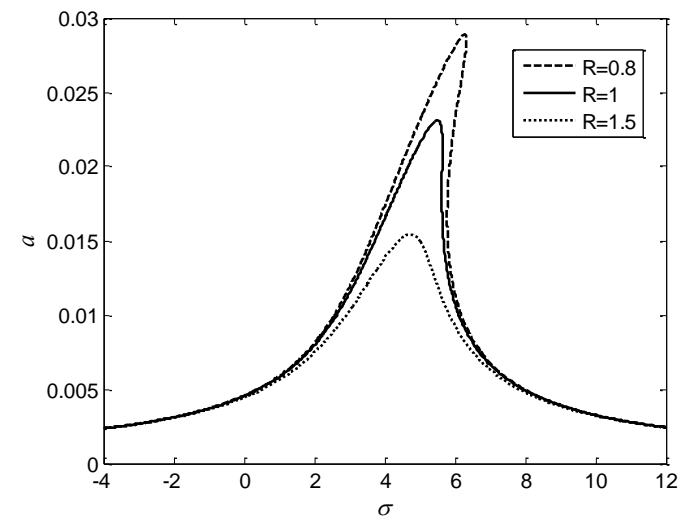

(b)

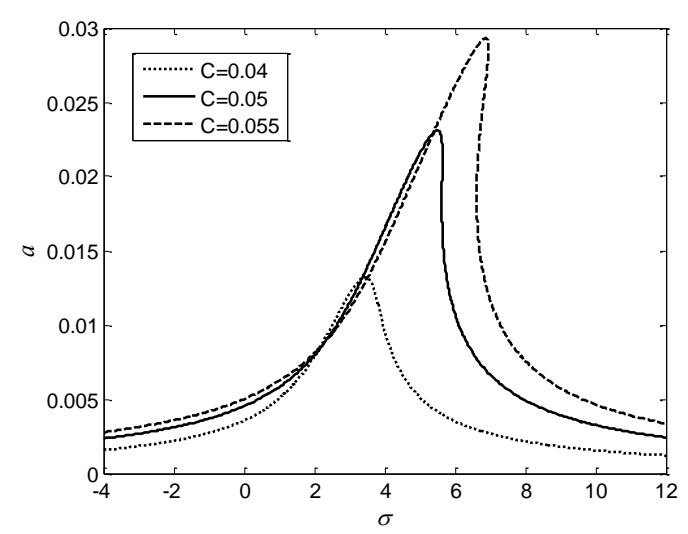

(d)

Figure 2. Amplitude frequency response curve

As can be seen in Figure 2, the general model of the sensor electronic components of RLC series circuit amplitude frequency response curve has the jump phenomena and hysteresis. They are the typical characteristics of nonlinear phenomena. Figure 2(a) displays when $E_{m}$ increases from $3.5 \mathrm{~V}$ to $4.5 \mathrm{~V}$, system resonance interval and amplitude value increase significantly, indicating system amplitude is sensitive to the change of the electromotive force. The charge circuit is reduced with the increase of resistance. System resonance interval and amplitude value decrease significantly and the nonlinear characteristic also weakens as shown in Figure 2(b). Figure2(c) displays system resonance interval obviously offset to the left when inductance increases from $0.4 \mathrm{H}$ to $0.8 \mathrm{H}$, and amplitude is significantly increased. It suggests that the system amplitude is very sensitive to the change of inductance. As shown in Figure 2(d) system resonance interval obviously offset to the right when capacitance increases from 0.05 to 0.055 , resonance range and the amplitude values are all increased. It suggests that the system amplitude is extremely sensitive to the change of 
capacitance. Electromotive force as a power element and resistance as the energy dissipation element have obvious effect to the RLC series circuit system vibration amplitude and resonance interval. Capacitance and inductance as energy storage have very significant effect to the RLC series circuit system of amplitude value when its numerical value changes a little.

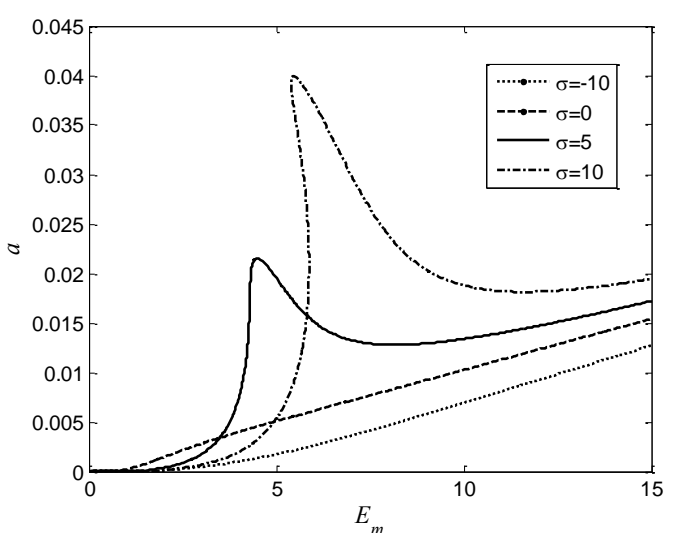

Figure 3. Amplitude-electromotive force response curves

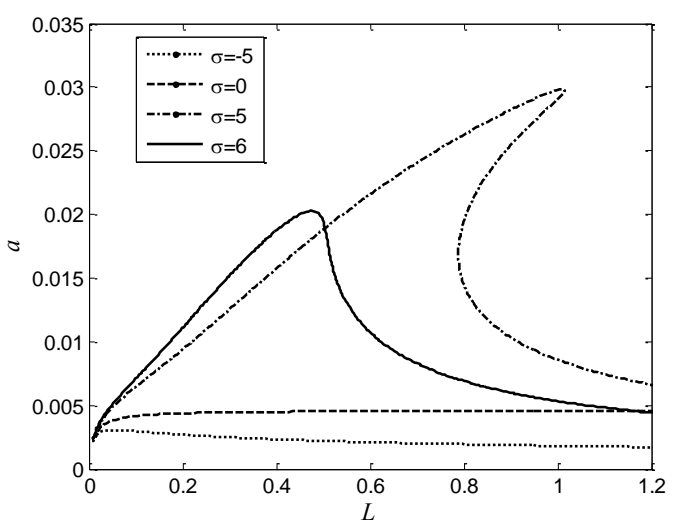

Figure 4. Amplitude - inductance response curves

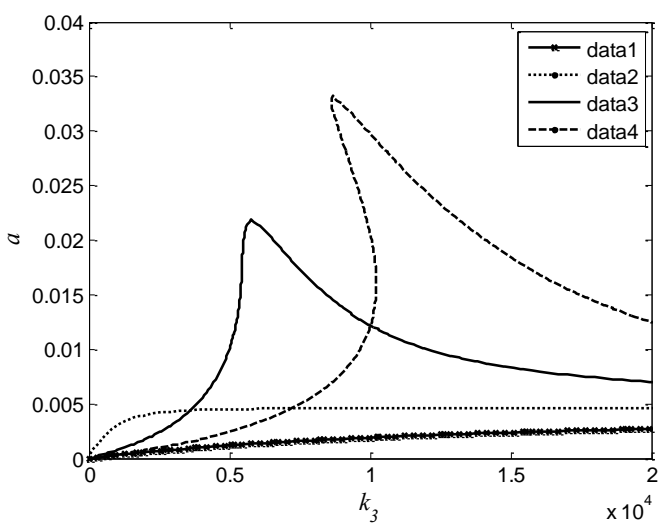

Figure 5. Amplitude - electric charge response curves 


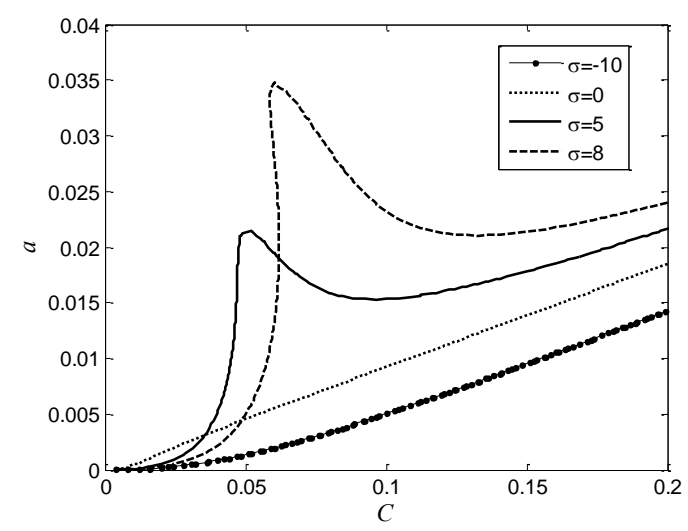

Figure 6. Amplitude - capacitance response curves

Figure 3 is amplitude-electromotive force response curves. The greater the detuning, the greater the amplitude of the RLC circuit when $\sigma>0$.Increasing the electromotive force, vibration amplitude jump to steadily increase at the same time. The sensor electronic components of RLC series circuit amplitude frequency response curve has the jump phenomena and hysteresis. The electromotive force increases with the increasing amplitude of the system with no jumping phenomenon and hysteresis when $\sigma \leq 0$. Figure 4 is amplitude - inductance response curves. The vibration amplitude of Electronic components of the RLC series circuit systems has hysteresis phenomenon when $\sigma>0$.Vibration amplitude tends to be stable when the inductance value is more than $1 \mathrm{H}$. RLC series resonant circuit system area is very sensitive to the inductance and it has both jumping and no jumping phenomena curves. Figure 5 is amplitude - electric charge response curves. System has hysteresis phenomena and finally tends to be stable under the effect of charge coefficient. Figure 6 is the curves of the influence of capacitance to the system amplitude. The vibration amplitude trends to stable increase with jumping and the increase of capacitance in a resonant response range. It should be pay more attention that the amplitude curves of two groups doesn't appear jumping phenomenon with the increase of capacitance. As seen from figure 3 to Figure 6, the resonant amplitude of sensor electronic components of the RLC series circuit systems has hysteresis phenomenon when $\sigma>0$ and while $\sigma \leq 0$ the jumping and hysteresis phenomena of resonant amplitude value disappear.

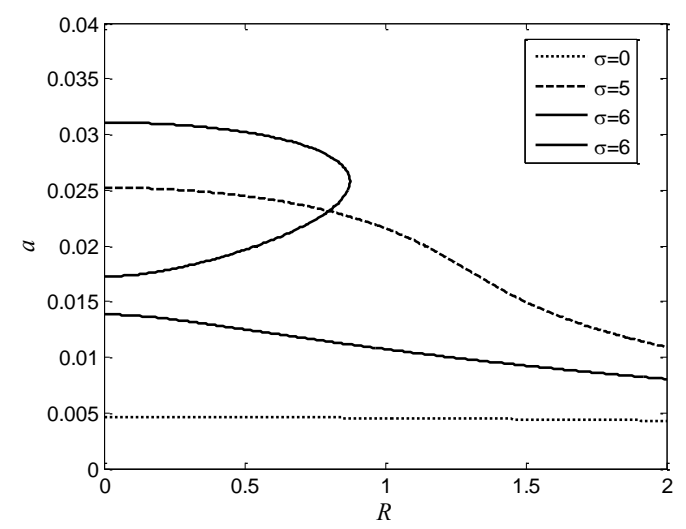

Figure 7. Amplitude- resistance sponse curves

Figure 7 is amplitude- resistance response curves. The greater the detuning, the amplitude of system hysteresis is more remarkable, and the jump phenomena appeared gradually. The resistance increases with the decreasing amplitude of the system, and finally the amplitude tends to be stable under the effect of charge coefficient. 


\section{Conclusions}

In view of the nonlinear characteristics of sensor components in practical engineering, the dynamic equation is established. From Figure 2 shows that the resistance can be inhibited amplitude, power supply can increase the amplitude, inductance, capacitance can make the jump and hysteresis phenomenon is more obvious. From figure 3- Figure 7 shows that meet certain conditions when $\sigma>0$, the sensor electronic components of the RLC circuit resonance phenomenon, and every element of RLC circuit are nonlinear, and the effect of parameter variations on nonlinear are significantly. Analysis of system parameters on the influence of the nonlinear vibration characteristics of sensor components, the conclusion can be developed for its security, reliability and sensitivity of design to provide the reference.

\section{Acknowledgements}

This research was financially supported by the Tangshan science technology bureau (15130262a), Tangshan university scientific research project(15011B).

\section{References}

1. Yu-Min XIANG. Journal of nonlinear dynamics, 1996, 3 (1) : 67-72.

2. Zhong-Yu YAO. Journal of Guangxi University ( Nat Sci Ed), 2001, 26(2): 145-149.

3. Dong-Sheng SUN, Jian-Song YANG. Journal of Hangzhou Teachers College( Natural Science), 2002, 1(1):31-33, 38.

4. M.I.YOUNIS, A.H.NAYFEH. Nonlinear Dynamics, 2003, 31:91-117.

5. Qing-Yi YANG, Wei-Ping ZHANG. Journal of Guangxi University (Natural Science Edition), 2013, 38 (6) : 1471-1475.

6. Hai-Ning SUN. Journal of Shanghai university, 2003, 9 (3) : 232-237

7. Ali Oksasoglu. IEEE Transaction on Circuit and Systems-I: Fundamental Theory and Application, 1994, 41( 10) : 669- 672.

8. S.K.CHAKRAVARTHY. IEE Proc-Electr. Power Appl, 1998, 145(6):585-592.

9. Zhi-An YANG, Yi-Hui CUI.. Pri mary Resonance Analysis of RLC Series Circuit with Resistance and Inductance Nonlinearity [J]. Journal of Tianjin University, 2007, 40(5):579-583.

10. Zhi-An YANG, Yi-Hui CUI. Chinese Journal Of Electron Devices, 2008, (3):988-991.

11. Yi-Hui CUI, Zhi-An YANG. Journal Of V Ibrat Ion And Shock, 2006, 25(4):76-77, 108, 177. 